\title{
Four-dimensional Einstein manifolds with Heisenberg symmetry
}

\author{
V. Cortés ${ }^{1}$ D $\cdot$ A. Saha ${ }^{1}$
}

Received: 25 May 2021 / Accepted: 5 August 2021 / Published online: 1 September 2021

(c) The Author(s) 2021

\begin{abstract}
We classify Einstein metrics on $\mathbb{R}^{4}$ invariant under a four-dimensional group of isometries including a principal action of the Heisenberg group. We consider metrics which are either Ricci-flat or of negative Ricci curvature. We show that all of the Ricci-flat metrics, including the simplest ones which are hyper-Kähler, are incomplete. By contrast, those of negative Ricci curvature contain precisely two complete examples: the complex hyperbolic metric and a metric of cohomogeneity one known as the one-loop deformed universal hypermultiplet.
\end{abstract}

Keywords Einstein metrics · Cohomogeneity one

MSC Classification 53C26

\section{Introduction}

It has been recently shown [4] that all the known homogeneous quaternionic Kähler manifolds of negative Ricci curvature with the exception of the simplest examples, the quaternionic hyperbolic spaces, admit a canonical deformation to a complete quaternionic Kähler manifold with an isometric action of cohomogeneity one. The deformation is a special case of what is known as the one-loop deformation [8]. The simplest example is a deformation of the complex hyperbolic plane known as the one-loop deformed universal hypermultiplet [1]. (The completeness requires the deformation parameter to be non-negative [2, Proposition 4].) Its isometry group is precisely $\mathrm{O}(2) \ltimes \mathrm{H}$, where $H$ is the three-dimensional Heisenberg group [4].

In this paper we determine all Einstein metrics which are invariant under the action of $\mathrm{SO}(2) \ltimes \mathrm{H}$ on $\mathbb{R}^{4}$. The symmetry assumption reduces the problem to the solution of a

V. Cortés

vicente.cortes@uni-hamburg.de

A. Saha

arpan.saha@uni-hamburg.de

1 Department of Mathematics, University of Hamburg, Bundesstraße 55, D-20146 Hamburg, Germany 
system of second-order ordinary differential equations for a pair of functions $a, b$, see (12)(14). The corresponding metrics are of the form

$$
g=d t^{2}+a(t)(d z+x d y-y d x)^{2}+b(t)\left(d x^{2}+d y^{2}\right) .
$$

The system admits solutions if and only if the Einstein constant $\Lambda$ is non-positive.

The Ricci-flat solutions include simple solutions of hyper-Kähler type (Proposition 4.3) as well as more complicated solutions (Proposition 4.5). They are all incomplete.

The solutions of negative Ricci curvature are described in Proposition 4.11. The stationary solutions are isometric to the complex hyperbolic plane (Proposition 4.1). The oneloop deformed universal hypermultiplet corresponds to a particular solution (Proposition 4.8), interpolating between a stationary solution and another fixed point of the flow defined by the subsystem (12)-(13).

The main result is that the only complete $\mathrm{SO}(2) \ltimes \mathrm{H}$-invariant Einstein metrics on $\mathbb{R}^{4}$ are the complex hyperbolic metric and its complete one-loop deformation (Theorem 4.13).

\section{Riemannian metrics with Heisenberg symmetry}

In this section we describe the class of metrics for which we will study the Einstein equation.

\subsection{The Heisenberg group}

Recall that the Heisenberg group $H$ is the unique simply connected nilpotent Lie group of dimension 3, up to isomorphism. We choose to realize it as $\mathbb{R}^{3}$ endowed with the following product:

$$
(x, y, z) \cdot(a, b, c)=(a+x, b+y, c+z+y a-x b)
$$

The advantage over other natural realizations (e.g. as the group of unipotent upper triangular matrices of rank 3$)$ is that the group $\operatorname{SL}(2, \mathbb{R})$ of unimodular transformations in the $(x, y)$-plane acts by automorphisms in these coordinates. This follows from Proposition 2.1. Abbreviating $v=(x, y)^{\top}$, we can write (1) more compactly as $\left(v_{1}, z_{1}\right) \cdot\left(v_{2}, z_{2}\right)=\left(v_{1}+v_{2}, z_{1}+z_{2}-\omega\left(v_{1}, v_{2}\right)\right)$, where $\omega=d x \wedge d y$. This implies the following.

Proposition 2.1 For any $A \in \mathrm{GL}(2, \mathbb{R})$ the transformation $(v, z) \mapsto(A v, z \operatorname{det} A)$ is an automorphism of $H$.

From (1) we can immediately read off that the left-invariant parallelization extending the standard basis at the neutral element is given by

$$
e_{1}=\partial_{x}+y \partial_{z}, \quad e_{2}=\partial_{y}-x \partial_{z}, \quad e_{3}=\partial_{z},
$$

with non-trivial structure constants determined by $\left[e_{1}, e_{2}\right]=-2 e_{3}$ or, equivalently, by $d e^{3}=2 e^{1} \wedge e^{2}$ in terms of the dual frame $\left(e^{i}\right)=\left(e_{i}^{*}\right)$.

Proposition 2.2 The isometry group $\operatorname{Isom}(H, g)$ of any left-invariant metric $g$ on $H$ is conjugate to $\mathrm{O}(2) \ltimes H$ in $\operatorname{Aut}(H) \ltimes H$. 
Proof By [10], the isometry group of $(H, g)$ is $\operatorname{Aut}(H, g) \ltimes H$, where $\operatorname{Aut}(H, g)=\operatorname{Aut}(H) \cap \operatorname{Isom}(H, g)$. Since every isometric automorphism preserves the center and its orthogonal complement, we see that, up to conjugation in $\operatorname{Aut}(H)$, we have the inclusion $\operatorname{Aut}(H, g) \subset \mathrm{O}(2)$. On the other hand, any orthogonal transformation of the orthogonal complement of the center extends uniquely to an isometric automorphism. This shows that, up to conjugation, $\operatorname{Isom}(H, g)=\mathrm{O}(2) \ltimes H$.

\subsection{Principal action of the Heisenberg group on $\mathbb{R}^{4}$}

Any complete Riemannian metric $g$ on $\mathbb{R}^{4}$ invariant under a principal action of the Heisenberg group $H$ can be brought to the form

$$
g=d t^{2}+g_{t},
$$

where $\mathbb{R}^{4}$ is identified with $\mathbb{R} \times H$ by an $H$-equivariant diffeomorphism and $g_{t}$ is a family of left-invariant metrics on $H$. This form is obtained by identifying the $H$-orbits by means of the normal geodesic flow, where $t$ corresponds to the arc length parameter along a normal geodesic.

The action of $\operatorname{Aut}(H) \ltimes H$ on $H$ trivially extends to $\mathbb{R}^{4}=\mathbb{R} \times H=\{(t, x, y, z)\}$.

Proposition 2.3 An H-invariant Riemannian metric $g=d t^{2}+g_{t}$ on $\mathbb{R}^{4}=\mathbb{R} \times H$ is invariant under $\mathrm{SO}(2) \subset \operatorname{Aut}(H)$ if and only if

$$
g_{t}=a(t)(d z+x d y-y d x)^{2}+b(t)\left(d x^{2}+d y^{2}\right),
$$

for some positive smooth functions $a, b \in C^{\infty}(\mathbb{R})$.

Proof We remark that (2) implies

$$
e^{1}=d x, \quad e^{2}=d y, \quad e^{3}=d z+x d y-y d x .
$$

Recall that, in terms of the coordinates $(t, x, y, z)$, the group $\mathrm{SO}(2)$ acts simply by rotations in the $(x, y)$-plane. The induced action on $H$-invariant one-forms on $\mathbb{R}^{4}$ is by rotations in the plane spanned by $e^{1}, e^{2}$, whereas the one-forms $e^{3}$ and $d t$ are invariant. As a consequence, $g_{t}$ (and hence $g$ ) is $\mathrm{SO}(2)$-invariant if and only of it is of the form (4).

Definition 2.4 $\mathrm{SO}(2) \ltimes H$-invariant metrics on $\mathbb{R}^{4}$, as described in Proposition 2.3, will be called metrics with maximal Heisenberg symmetry.

The main problem studied in this paper is the following.

Problem 2.5 Determine all Einstein metrics on $\mathbb{R}^{4}$ with maximal Heisenberg symmetry.

The following consequence of Proposition 2.3 is used in the calculations of the connection and the curvature in the next section. Note also that the map $(t, x, y, z) \mapsto(t, y,-x, z)$ is an isometry (in the group $\mathrm{SO}(2))$, which can be also used for that purpose. 
Corollary 2.6 Any metric $g$ with maximal Heisenberg symmetry on $\mathbb{R}^{4}$ is $\mathrm{O}(2)$-invariant, that is not only $\mathrm{SO}(2)$-invariant but, in addition, invariant under the involution $\sigma:(t, x, y, z) \mapsto(t, y, x,-z)$. The surface

$$
\Sigma=\left(\mathbb{R}^{4}\right)^{\sigma}=\left\{p \in \mathbb{R}^{4} \mid \sigma(p)=p\right\}=\{(t, x, x, 0) \mid t, x \in \mathbb{R}\}
$$

is totally geodesic and induces an H-invariant foliation of $\mathbb{R}^{4}$ by totally geodesic surfaces. The leaf through a point $p_{0}=\left(t_{0}, x_{0}, y_{0}, z_{0}\right)$ is given by

$$
\Sigma_{p_{0}}=\left(x_{0}, y_{0}, z_{0}\right) \cdot \Sigma=\left\{(t, x, y, z) \mid x-y=x_{0}-y_{0}, \quad z=\left(y_{0}-x_{0}\right)\left(x-x_{0}\right)+z_{0}\right\} .
$$

\section{Einstein equation for metrics with maximal Heisenberg symmetry}

In this section we determine the system of ordinary differential equations satisfied by Einstein metrics with maximal Heisenberg symmetry. First we compute the Levi-Civita connection and Ricci curvature of such metrics.

Throughout this section

$$
g=d t^{2}+a(t)(d z+x d y-y d x)^{2}+b(t)\left(d x^{2}+d y^{2}\right)
$$

denotes a metric with maximal Heisenberg symmetry on $\mathbb{R}^{4}$.

\subsection{Connection and Ricci curvature}

Proposition 3.1 The Levi-Civita connection $\nabla$ of a metric (8) with maximal Heisenberg symmetry is given by

$$
\begin{aligned}
& \nabla_{\partial_{t}} \partial_{t}=0, \quad \nabla_{\partial_{t}} \partial_{z}=\frac{1}{2}(\ln a)^{\prime} \partial_{z}, \quad \nabla_{\partial_{z}} \partial_{z}=-\frac{1}{2} a^{\prime} \partial_{t}, \\
& \nabla_{\partial_{t}} \partial_{x}=\frac{y}{2}\left(\ln \frac{b}{a}\right)^{\prime} \partial_{z}+\frac{1}{2}(\ln b)^{\prime} \partial_{x}, \quad \nabla_{\partial_{t}} \partial_{y}=\frac{x}{2}\left(\ln \frac{a}{b}\right)^{\prime} \partial_{z}+\frac{1}{2}(\ln b)^{\prime} \partial_{y}, \\
& \nabla_{\partial_{z}} \partial_{x}=\frac{1}{2} a^{\prime} y \partial_{t}-\frac{a}{b} x \partial_{z}+\frac{a}{b} \partial_{y}, \quad \nabla_{\partial_{z}} \partial_{y}=-\frac{1}{2} a^{\prime} x \partial_{t}-\frac{a}{b} y \partial_{z}-\frac{a}{b} \partial_{x}, \\
& \nabla_{\partial_{x}} \partial_{x}=-\frac{1}{2}\left(a^{\prime} y^{2}+b^{\prime}\right) \partial_{t}+2 \frac{a}{b} x y \partial_{z}-2 \frac{a}{b} y \partial_{y}, \\
& \nabla_{\partial_{y} \partial_{y}}=-\frac{1}{2}\left(a^{\prime} x^{2}+b^{\prime}\right) \partial_{t}-2 \frac{a}{b} x y \partial_{z}-2 \frac{a}{b} x \partial_{x}, \\
& \nabla_{\partial_{x}} \partial_{y}=\frac{1}{2} a^{\prime} x y \partial_{t}+\frac{a}{b}\left(y^{2}-x^{2}\right) \partial_{z}+\frac{a}{b} y \partial_{x}+\frac{a}{b} x \partial_{y} .
\end{aligned}
$$

Proposition 3.2 The Ricci curvature $\operatorname{Ric}_{g}=\sum R_{i j} d x^{i} d x^{j}$, of $g$ is given in the coordinates $\left(x^{0}, x^{1}, x^{2}, x^{3}\right)=(t, x, y, z)$ by 


$$
\begin{aligned}
& R_{00}=-\left(\frac{1}{4}\left((\ln a)^{\prime}\right)^{2}+\frac{1}{2}\left((\ln b)^{\prime}\right)^{2}+\frac{1}{2}(\ln a)^{\prime \prime}+(\ln b)^{\prime \prime}\right) g_{00}, \\
& R_{11}=-\frac{1}{2}\left(a^{\prime \prime} y^{2}+b^{\prime \prime}\right)-\frac{1}{4}(\ln a)^{\prime} b^{\prime}+2 \frac{a^{2}}{b^{2}} y^{2}+\frac{y^{2}}{4}(\ln a)^{\prime} a^{\prime}-\frac{1}{2} a^{\prime} y^{2}(\ln b)^{\prime}-2 \frac{a}{b}, \\
& R_{22}=-\frac{1}{2}\left(a^{\prime \prime} x^{2}+b^{\prime \prime}\right)-\frac{1}{4}(\ln a)^{\prime} b^{\prime}+2 \frac{a^{2}}{b^{2}} x^{2}+\frac{x^{2}}{4}(\ln a)^{\prime} a^{\prime}-\frac{1}{2} a^{\prime} x^{2}(\ln b)^{\prime}-2 \frac{a}{b}, \\
& R_{33}=\left(-\frac{a^{\prime \prime}}{2 a}+\frac{\left(a^{\prime}\right)^{2}}{4 a^{2}}-\frac{a^{\prime} b^{\prime}}{2 a b}+2 \frac{a}{b^{2}}\right) g_{33}, \\
& R_{12}=\frac{1}{2} a^{\prime \prime} x y-\frac{\left(a^{\prime}\right)^{2}}{4 a} x y-2\left(\frac{a}{b}\right)^{2} x y+\frac{1}{2} a^{\prime}(\ln b)^{\prime} x y, \\
& R_{13}=\frac{1}{2} a^{\prime \prime} y+\frac{y}{4} a^{\prime}\left(\ln \frac{b}{a}\right)^{\prime}-2\left(\frac{a}{b}\right)^{2} y+\frac{1}{4} a^{\prime}(\ln b)^{\prime} y, \\
& R_{23}=-\left(\frac{1}{2} a^{\prime \prime} x+\frac{x}{4} a^{\prime}\left(\ln \frac{b}{a}\right)^{\prime}-2\left(\frac{a}{b}\right)^{2} x+\frac{1}{4} a^{\prime}(\ln b)^{\prime} x\right), \\
& R_{01}=0=g_{01}, \quad R_{02}=0=g_{02}, \quad R_{03}=0=g_{03} .
\end{aligned}
$$

\subsection{Einstein equation}

Corollary 3.3 A metric (8) with maximal Heisenberg symmetry is Einstein, $\operatorname{Ric}_{g}=\Lambda g$, with constant $\Lambda$ if and only if

$$
\begin{aligned}
& \frac{1}{4}\left((\ln a)^{\prime}\right)^{2}+\frac{1}{2}\left((\ln b)^{\prime}\right)^{2}+\frac{1}{2}(\ln a)^{\prime \prime}+(\ln b)^{\prime \prime}=\frac{a^{\prime \prime}}{2 a}-\frac{\left(a^{\prime}\right)^{2}}{4 a^{2}}+\frac{a^{\prime} b^{\prime}}{2 a b}-\frac{2 a}{b^{2}}=-\Lambda, \\
& \frac{1}{2} \frac{b^{\prime \prime}}{b}+\frac{1}{4}(\ln a)^{\prime}(\ln b)^{\prime}+\frac{2 a}{b^{2}}=-\Lambda .
\end{aligned}
$$

Corollary 3.4 The metric $g$ is Einstein with constant $\Lambda$ if and only if the functions $\lambda=(\ln a)^{\prime}$ and $\mu=(\ln b)^{\prime}$ satisfy the following overdetermined system of ordinary differential equations:

$$
\begin{aligned}
& 2 \lambda^{\prime}+4 \mu^{\prime}+\lambda^{2}+2 \mu^{2}+4 \Lambda=0, \\
& 2 \lambda^{\prime}+\lambda^{2}+2 \lambda \mu-\frac{8 a}{b^{2}}+4 \Lambda=0, \\
& 2 \mu^{\prime}+2 \mu^{2}+\lambda \mu+\frac{8 a}{b^{2}}+4 \Lambda=0 .
\end{aligned}
$$

The system is equivalent to

$$
\begin{gathered}
2 \lambda^{\prime}=-\left(\lambda^{2}+2 \mu^{2}+6 \lambda \mu+12 \Lambda\right), \\
2 \mu^{\prime}=3 \lambda \mu+4 \Lambda,
\end{gathered}
$$




$$
0=\mu^{2}+2 \lambda \mu+\frac{4 a}{b^{2}}+4 \Lambda
$$

Proof The first system is obtained by substitution of the variables. Adding the equations (10) and (11) we obtain

$$
2 \lambda^{\prime}+2 \mu^{\prime}+\lambda^{2}+2 \mu^{2}+3 \lambda \mu+8 \Lambda=0 .
$$

Using this equation we eliminate, respectively, $\mu^{\prime}$ and $\lambda^{\prime}$ from (9) arriving at (12) and (13). Finally, comparing (11) with (13) yields (14).

\section{Solutions}

\subsection{Classification of stationary solutions}

We call a solution $(a(t), b(t))$ of the ode system (12)-(14) stationary if $\lambda^{\prime}=\mu^{\prime}=0$.

Proposition 4.1 The stationary solutions $(a, b)$ of the Einstein equations (12)-(14) are given by

$$
a=-\frac{\Lambda}{6} b^{2}, \quad b=C e^{\mu t},
$$

where $\mu \neq 0$ and $C>0$ are constants. The corresponding Einstein manifold $\left(\mathbb{R}^{4}, g\right)$ is isometric to the complex hyperbolic plane of Einstein constant $\Lambda=-\frac{3}{2} \mu^{2}<0$.

Proof Since $\lambda$ and $\mu$ are constant for stationary solutions, we see from (14) that the function $a / b^{2}$ is constant and, hence,

$$
\lambda-2 \mu=0 .
$$

Inserting this into (12)-(13) we obtain

$$
\Lambda=-\frac{3}{2} \mu^{2}
$$

and (14) then yields

$$
\Lambda=-\frac{6 a}{b^{2}} .
$$

This shows that $\Lambda<0$ and, hence, $\mu \neq 0$. The above metrics are all homothetic to

$$
d t^{2}+e^{4 t}(d z+x d y-y d x)^{2}+e^{2 t}\left(d x^{2}+d y^{2}\right),
$$

by Remark 4.2. This is the complex hyperbolic metric of holomorphic sectional curvature -4 (i.e. $\Lambda=-6$ ) written as a left-invariant metric on the simply transitive solvable Iwasawa subgroup of its group of holomorphic isometries $\operatorname{PSU}(1,2)$.

Remark 4.2 The two parameters of the solution (15) correspond to the freedom to reparametrize the $t$-variable by an affine transformation. In fact, a transformation of the 
coordinates $(t, x, y, z)$ by a pure translation in $t$ yields another stationary solution but with another $C$-parameter, whereas rescaling of the $t$-variable in the coordinate system yields an Einstein metric which, up to a constant conformal factor, is a stationary solution in our class (8), the latter with another $\mu$-parameter.

Note that the transformation $(a, b) \mapsto(\tilde{a}, \tilde{b})$, where

$$
\tilde{a}(t):=\frac{a(k t)}{k^{2}}, \quad \tilde{b}(t):=\frac{b(k t)}{k^{2}}, \quad k \in \mathbb{R} \backslash\{0\},
$$

maps arbitrary solutions of (12)-(14) to (homothetic) solutions. In this way one can always normalize a given solution with $\Lambda<0$ such that $\Lambda=-6$.

\subsection{Ricci-flat solutions}

Proposition 4.3 There exist solutions $(a, b)$ of the Einstein equations (12)-(14) with $\lambda=\frac{\ell}{t}$ and $\mu=\frac{m}{t}, \ell, m \in \mathbb{R}$. They are all hyper-Kähler and of the form

$$
a(t)=a_{1}|t|^{-2 / 3}, \quad b(t)=b_{1}|t|^{2 / 3},
$$

where $a_{1}, b_{1}$ are positive constants such that $a_{1} / b_{1}^{2}=1 / 9$. The maximal domains of definition of these (incomplete) metrics are $\mathbb{R}_{>0} \times \mathbb{R}^{3}$ and $\mathbb{R}_{<0} \times \mathbb{R}^{3}$.

Proof Under the ansatz $\lambda=\frac{\ell}{t}$ and $\mu=\frac{m}{t}$, the equation (13) implies that $\Lambda=0$. The equations (12)-(13) can then be easily solved in terms of $(\ell, m)$. We find that $(\ell, m)$ is one of the following: $\left(-\frac{2}{3}, \frac{2}{3}\right),\left(-\frac{2}{3}, \frac{4}{3}\right),(2,0),(0,0)$. However, the last three cases are clearly inconsistent with equation (14). (The case $(0,0)$ is also excluded, because such a solution would be stationary contrary to Proposition 4.1.) So we are left with studying equation (14) in the case $(\ell, m)=\left(-\frac{2}{3}, \frac{2}{3}\right)$. Inserting $a=a_{1}|t|^{\ell}=a_{1}|t|^{-2 / 3}$ and $b=b_{1}|t|^{m}=b_{1}|t|^{2 / 3}$ we obtain $a_{1} / b_{1}^{2}=1 / 9$.

The given metric can now be explicitly shown to be hyper-Kähler. Consider the following two-forms:

$$
\begin{aligned}
\omega_{1} & =\sqrt{a} d t \wedge(d z+x d y-y d x)+\operatorname{sign}(t) b d x \wedge d y \\
& =\sqrt{a_{1}}|t|^{-1 / 3} d t \wedge(d z+x d y-y d x)+3 \operatorname{sign}(t) \sqrt{a_{1}}|t|^{2 / 3} d x \wedge d y, \\
\omega_{2} & =\sqrt{b} d t \wedge d y+\operatorname{sign}(t) \sqrt{a b}(d z+x d y-y d x) \wedge d x \\
& =\sqrt{b_{1}}|t|^{1 / 3} d t \wedge d y+\operatorname{sign}(t) \sqrt{a_{1} b_{1}}(d z+x d y-y d x) \wedge d x, \\
\omega_{3} & =\sqrt{b} d t \wedge d x+\operatorname{sign}(t) \sqrt{a b} d y \wedge(d z+x d y-y d x) \\
& =\sqrt{b_{1}}|t|^{1 / 3} d t \wedge d x+\operatorname{sign}(t) \sqrt{a_{1} b_{1}} d y \wedge(d z+x d y-y d x) .
\end{aligned}
$$

These are (anti-)self-dual and closed, and so form a hyper-Kähler structure.

Remark 4.4 The incomplete hyper-Kähler metrics described in Proposition 4.3 are all homothetic to a single metric, compare (17). The metric can be obtained from a GibbonsHawking ansatz and admits a conformal rescaling to a complete left-invariant metric on the solvable Iwasawa subgroup of $S U(1,2)$ [5, Section 3.2.2]. The metric also appears in the 
study of collapsing hyper-Kähler metrics on K3 surfaces [7], as we learned from Simon Salamon [9].

Proposition 4.5 If $(a, b)$ is a Ricci-flat solution of the Einstein equations (12)-(14) not isometric to (18), then the associated functions $\lambda$ and $\mu$ satisfy

$$
\frac{2}{3 \mu}\left(\mp_{2} F_{1}\left(-\frac{3}{4}, \frac{1}{2} ; \frac{1}{4} ;-C|\mu|^{4 / 3}\right)+1\right)=t-t_{0}, \quad \lambda= \pm \frac{\mu \sqrt{1+C|\mu|^{4 / 3}}}{1 \mp \sqrt{1+C|\mu|^{4 / 3}}},
$$

where $C>0$ and $t_{0}$ are constants and ${ }_{2} F_{1}$ is the hypergeometric function. The corresponding metrics are incomplete. In fact, the maximal domains of definition of these metrics are

$$
\begin{aligned}
& ]-\infty, t_{0}-\frac{2 C^{3 / 4}}{3 \sqrt{\pi}} \Gamma\left(\frac{1}{4}\right) \Gamma\left(\frac{5}{4}\right)\left[\times \mathbb{R}^{3},\right] t_{0}-\frac{2 C^{3 / 4}}{3 \sqrt{\pi}} \Gamma\left(\frac{1}{4}\right) \Gamma\left(\frac{5}{4}\right), t_{0}\left[\times \mathbb{R}^{3},\right. \\
& ] t_{0}+\frac{2 C^{3 / 4}}{3 \sqrt{\pi}} \Gamma\left(\frac{1}{4}\right) \Gamma\left(\frac{5}{4}\right),+\infty\left[\times \mathbb{R}^{3},\right] t_{0}, t_{0}+\frac{2 C^{3 / 4}}{3 \sqrt{\pi}} \Gamma\left(\frac{1}{4}\right) \Gamma\left(\frac{5}{4}\right)\left[\times \mathbb{R}^{3} .\right.
\end{aligned}
$$

Proof Setting $\Lambda=0$ in (12)-(14) gives us

$$
\begin{gathered}
2 \lambda^{\prime}=-\left(\lambda^{2}+6 \lambda \mu+2 \mu^{2}\right), \\
2 \mu^{\prime}=3 \lambda \mu, \\
-\frac{4 a}{b^{2}}=\mu(\mu+2 \lambda) .
\end{gathered}
$$

On a domain where $\mu$ and $\mu+2 \lambda$ are non-vanishing, (21) and (22) imply

$$
\frac{d(\mu(\mu+2 \lambda))}{\mu(\mu+2 \lambda)}=\lambda d t-2 \mu d t
$$

Integrating and then exponentiating both sides gives

$$
\mu^{2}+2 \lambda \mu=-\frac{4 k a}{b^{2}},
$$

where $k$ is a nonzero constant of integration. Notice, however, that given $\lambda=(\ln a)^{\prime}$ and $\mu=(\ln b)^{\prime}$, the positive functions $a$ and $b$ are determined only up to overall positive constant factors. Thus, the constant $k$ may be absorbed into this indeterminacy so that the constraint (23) is satisfied, provided that $k>0$.

In particular, this argument fails when either $\mu$ or $\mu+2 \lambda$ vanish. In fact, (23) then necessarily means that $a$ vanishes, which is not allowed. Therefore, the constraint amounts to stipulating that $\mu$ and $\mu+2 \lambda$ are non-vanishing on the domain of definition and of opposite sign.

We will now describe general non-stationary solutions of (21) and (22). If $\lambda$ is a constant function, then so is $\mu$. We may thus assume that $\lambda$ is not everywhere 0 . As $\mu$ is constrained to be non-vanishing, $\mu^{\prime}=\frac{3}{2} \lambda \mu$ must also be non-vanishing on the (open) complement of the vanishing set of $\lambda$. On this open set, we may regard $t$, and hence $\lambda(t)$, as an implicit function of $\mu$. Then $\lambda$ satisfies the following ode: 


$$
\frac{d \lambda}{d \mu}=\frac{\lambda^{\prime}}{\mu^{\prime}}=-\frac{\lambda^{2}+6 \lambda \mu+2 \mu^{2}}{3 \lambda \mu}
$$

Define a function $v$ by $\lambda=\mu \nu$. Substituting this into the above equation and rearranging the terms gives us

$$
\mu \frac{d v}{d \mu}=-\frac{4 v^{2}+6 v+2}{3 v}
$$

There are two cases to be considered now: either the numerator of the right-hand side is identically zero or it is not.

Let us suppose the first case, that is

$$
4 v^{2}+6 v+2=2(v+1)(2 v+1)=0 .
$$

Then $v$ takes the value -1 or $-\frac{1}{2}$. Note that if $v=-\frac{1}{2}$, then

$$
\mu+2 \lambda=\mu+2 \mu \nu=0 .
$$

So this is not allowed, and $\nu$ must necessarily be -1 . Thus, $\lambda=-\mu$ and $\mu^{\prime}=\frac{3}{2} \lambda \mu=-\frac{3}{2} \mu^{2}$. Up to constant shifts in $t$, this gives the same solution as in (18), and so is excluded as well.

So $4 v^{2}+6 v+2$ cannot be identically zero. On the complement of its vanishing set, we may separate the variables and integrate to obtain

$$
\ln |v+1|-\frac{1}{2} \ln |2 v+1|=-\frac{2}{3} \ln |\mu|+\text { const. }
$$

Multiplying by -2 throughout and then exponentiating both sides gives us

$$
\frac{2 v+1}{(v+1)^{2}}=-C|\mu|^{4 / 3},
$$

where $C$ is some nonzero constant. Then solving for $v$, we get

$$
\nu= \pm \frac{\sqrt{1+C|\mu|^{4 / 3}}}{1 \mp \sqrt{1+C|\mu|^{4 / 3}}} .
$$

Thus, $\lambda$ as a function of $\mu$ is given by

$$
\lambda=\mu \nu= \pm \frac{\mu \sqrt{1+C|\mu|^{4 / 3}}}{1 \mp \sqrt{1+C|\mu|^{4 / 3}}} .
$$

To now obtain $\mu$ as function of $t$, we substitute the above expression into (22):

$$
2 \mu^{\prime}= \pm \frac{3 \mu^{2} \sqrt{1+C|\mu|^{4 / 3}}}{1 \mp \sqrt{1+C|\mu|^{4 / 3}}} .
$$

Separating the variables and integrating gives us the equation

$$
\frac{2}{3 \mu}\left(\mp_{2} F_{1}\left(-\frac{3}{4}, \frac{1}{2} ; \frac{1}{4} ;-C|\mu|^{4 / 3}\right)+1\right)=t-t_{0},
$$


where $t_{0}$ is a constant of integration. To see this, we remark that the hypergeometric function ${ }_{2} F_{1}(a, b ; c ; x)$, for $c=a+1, a \neq 0$, is related to the incomplete beta function $B_{x}(a, 1-b)$ by

$$
B_{x}(a, 1-b)={ }_{2} F_{1}(a, b ; a+1 ; x) \frac{x^{a}}{a} .
$$

This implies that ${ }_{2} F_{1}(a, b ; a+1 ; x)$ satisfies the first-order ode $F^{\prime}(x)=\frac{a\left((1-x)^{-b}-F(x)\right)}{x}$, which leads to (25).

To determine the maximal domains of definition of the metric, we determine the values of $t$ for which either at least one of $\lambda$ and $\mu$ becomes infinite or for which we have $\mu(\mu+2 \lambda)=0$.

We find that for the upper branch of the solution, that is

$$
\frac{2}{3 \mu}\left(-{ }_{2} F_{1}\left(-\frac{3}{4}, \frac{1}{2} ; \frac{1}{4} ;-C|\mu|^{4 / 3}\right)+1\right)=t-t_{0}, \quad \lambda=\frac{\mu \sqrt{1+C|\mu|^{4 / 3}}}{1-\sqrt{1+C|\mu|^{4 / 3}}},
$$

taking the limit $\mu \rightarrow 0^{ \pm}$gives us $t=t_{0}$ and $\lambda \rightarrow \mp \infty$. By contrast, on the lower branch of the solution, that is

$$
\frac{2}{3 \mu}\left(+{ }_{2} F_{1}\left(-\frac{3}{4}, \frac{1}{2} ; \frac{1}{4} ;-C|\mu|^{4 / 3}\right)+1\right)=t-t_{0}, \quad \lambda=-\frac{\mu \sqrt{1+C|\mu|^{4 / 3}}}{1+\sqrt{1+C|\mu|^{4 / 3}}},
$$

the limit $\mu \rightarrow 0^{ \pm}$gives $t \rightarrow \pm \infty$ and $\lambda \rightarrow 0$.

Meanwhile, setting $\mu+2 \lambda=0$ is the same as setting $v=\frac{\lambda}{\mu}=-\frac{1}{2}$, giving us

$$
-\frac{1}{2}= \pm \frac{\sqrt{1+C|\mu|^{4 / 3}}}{1 \mp \sqrt{1+C|\mu|^{4 / 3}}}
$$

This is solved only by $\mu=0$ on the lower branch of the solution, and therefore for no finite value of $t$.

In the case that $C$ is positive, we can take the limit $\mu \rightarrow \pm \infty$ to obtain on the upper branch

$$
t=t_{0} \mp \frac{2 C^{3 / 4}}{3 \sqrt{\pi}} \Gamma\left(\frac{1}{4}\right) \Gamma\left(\frac{5}{4}\right), \quad \lambda \rightarrow \mp \infty
$$

and on the lower branch

$$
t=t_{0} \pm \frac{2 C^{3 / 4}}{3 \sqrt{\pi}} \Gamma\left(\frac{1}{4}\right) \Gamma\left(\frac{5}{4}\right), \quad \lambda \rightarrow \mp \infty .
$$

Note that the above cases automatically take care of the limits in which $\lambda$ becomes infinite. The above limits are obtained by specializing the asymptotics for $|x| \rightarrow \infty$ of the hypergeometric function $F(x)={ }_{2} F_{1}(a, b ; c ; x)$ for $a-b \notin \mathbb{Z}$ to $(a, b, c)=\left(-\frac{3}{4}, \frac{1}{2} ; \frac{1}{4}\right)$ :

$$
F(x) \sim \frac{\Gamma(b-a) \Gamma(c)}{\Gamma(b) \Gamma(c-a)}(-x)^{-a}+\frac{\Gamma(a-b) \Gamma(c)}{\Gamma(a) \Gamma(-b)}(-x)^{-b} .
$$


Putting everything together, we find that the maximal domains of definition for $t$ are the open intervals $]-\infty, t_{0}$ [ and $] t_{0},+\infty[$ when $C<0$, and the following open intervals when $C>0$ :

$$
\begin{aligned}
& -\infty, t_{0}-\frac{2 C^{3 / 4}}{3 \sqrt{\pi}} \Gamma\left(\frac{1}{4}\right) \Gamma\left(\frac{5}{4}\right)[,] t_{0}-\frac{2 C^{3 / 4}}{3 \sqrt{\pi}} \Gamma\left(\frac{1}{4}\right) \Gamma\left(\frac{5}{4}\right), t_{0}[, \\
& ] t_{0}+\frac{2 C^{3 / 4}}{3 \sqrt{\pi}} \Gamma\left(\frac{1}{4}\right) \Gamma\left(\frac{5}{4}\right),+\infty[,] t_{0}, t_{0}+\frac{2 C^{3 / 4}}{3 \sqrt{\pi}} \Gamma\left(\frac{1}{4}\right) \Gamma\left(\frac{5}{4}\right)[.
\end{aligned}
$$

Now that we have described all the solutions to the ode system, we check which of them satisfy the sign constraint $\mu(\mu+2 \lambda)<0$ to determine which of them correspond to Riemanninan metrics. Dividing the sign constraint by $\mu^{2}>0$, we find that it is equivalent to $2 v+1<0$. From (24) we see that this happens precisely when $C>0$.

Remark 4.6 By taking $t$ purely imaginary and the integration constant $t_{0}$ complex, one can similarly describe Ricci-flat Lorentzian metrics of the form

$$
g=-d t^{2}+a(t)(d z+x d y-y d x)^{2}+b(t)\left(d x^{2}+d y^{2}\right)
$$

from solutions of (25) with $C<0$. As in the Riemannian case, these are $\mathrm{O}(2) \ltimes H$-invariant. Lorentzian solutions of the Einstein equations invariant under a principal action of a three-dimensional Lie group with space-like orbits have been studied as cosmological models in general relativity, see [6].

Remark 4.7 The limit $C \rightarrow+\infty$ is in fact well defined. In this limit, (19) becomes

$$
\frac{2}{3 \mu}=t-t_{0}, \quad \lambda=-\mu
$$

A constant shift $t \mapsto t-t_{0}$ then reproduces the solution (18).

\subsection{The one-loop deformed universal hypermultiplet}

In this section we exhibit a family of solutions of the Einstein equations (12)-(14) with $\Lambda=-6$ depending on a real parameter $c$. The solution is stationary only for $c=0$, in which case the metric is the complex hyperbolic metric (16).

Let $c$ be a real constant and let $I$ be a connected component of the set

$$
\{\rho \in \mathbb{R} \mid \rho \neq 0, \rho+c>0 \text { and } \rho+2 c>0\} .
$$

Let $\rho: J \stackrel{\sim}{\rightarrow} I, t \mapsto \rho(t)$, be a (maximal) solution of the differential equation

$$
\rho^{\prime}(t)=2 \rho(t) \sqrt{\frac{\rho(t)+c}{\rho(t)+2 c}}
$$

which is defined on some interval $J$ and has the interval $I$ as its range. The functions 


$$
a(t)=\frac{\rho(t)+c}{4 \rho(t)^{2}(\rho(t)+2 c)} \quad \text { and } \quad b(t)=\frac{\rho(t)+2 c}{2 \rho(t)^{2}}
$$

are positive on their domain $J$.

Recall (see Remark 4.2) that the Einstein constant $\Lambda$ of a solution of (12)-(14) is either zero or the metric can be rescaled such that $\Lambda$ is any constant negative number.

Proposition 4.8 The functions $a(t)$ and $b(t)$ defined by (28) and (27) constitute a oneparameter family of solutions of the Einstein equations (12)-(14) with $\Lambda=-6$. The corresponding metrics are complete if and only if $c>0$ and $I=\{\rho \mid \rho>0\}$.

Proof Writing the metric $g=d t^{2}+a(t)(d z+x d y-y d x)^{2}+b(t)\left(d x^{2}+d y^{2}\right)$ in terms of the coordinates $(\rho, x, y, z)$ instead of $(t, x, y, z)$ shows that it coincides with the one-loop deformed universal hypermultiplet metric, as given in equation (1.1) of [3]. (For the physical origins and significance of this metric see [1,8].) The metric is not only Einstein of Einstein constant -6 but is half conformally flat and is complete if and only if $c>0$ and $I=\{\rho \mid \rho>0\}$, see [2]. Moreover, it was shown in [4, Theorem 4.5] that for $c \neq 0$ the metric has the isometry group $\mathrm{O}(2) \rtimes H$, where $H$ denotes the Heisenberg group. (For $c=0$ the metric is the complex hyperbolic metric discussed in Sect. 4.1.) This proves Proposition 4.8 .

Alternatively, one can check directly that the functions $a(t)$ and $b(t)$ solve the system (12)-(14). In fact, the equations (13) and (14) are easily checked and (14) implies (12) on the set where $\mu \neq 0$. The latter is shown by differentiating (14) and using the simple equation

$$
\left(\frac{a}{b^{2}}\right)^{\prime}=\frac{a}{b^{2}}(\lambda-2 \mu)
$$

A short calculation shows that for the above functions $a$ and $b$, the function $\mu$ vanishes only if $c<0$ and $-4 c \in I$. In that case, the zero is at $\rho=-4 c$, i.e. at $t=\rho^{-1}(-4 c)$. The equation (12) follows by continuity, since the complement of the zero set is dense.

Proposition 4.9 A solution $(\lambda(t), \mu(t))$ of the Einstein equations (12)-(14) corresponding to the one-loop deformed universal hypermultiplet satisfies the following polynomial constraint of degree 4:

$$
P(\lambda, \mu):=(\lambda+\mu)^{3} \mu-4\left(3 \lambda^{2}+18 \lambda \mu+11 \mu^{2}\right)+512=0 .
$$

Proof From (28), we obtain the following parametrisation of $\lambda$ and $\mu$ in terms of $\rho$ :

$$
\begin{gathered}
\lambda=(\ln a)^{\prime}=\frac{\rho^{\prime}}{\rho}\left(\frac{\rho}{\rho+c}-2-\frac{\rho}{\rho+2 c}\right), \\
\mu=(\ln b)^{\prime}=\frac{\rho^{\prime}}{\rho}\left(\frac{\rho}{\rho+2 c}-2\right)=-\frac{\rho^{\prime}}{\rho}\left(\frac{\rho+4 c}{\rho+2 c}\right) .
\end{gathered}
$$

In particular, on a domain where $\mu$ is non-vanishing, we have $\frac{4 c}{\rho}+1 \neq 0$ and we can combine the above equations to get 


$$
\frac{\lambda+\mu}{\mu}=\left(\frac{\rho}{\rho+c}-4\right)\left(\frac{\rho}{\rho+2 c}-2\right)^{-1}=2+\left(\frac{4 c}{\rho}+1\right)^{-1}\left(\frac{c}{\rho}+1\right)^{-1} .
$$

By subtracting 2 throughout, we see that this implies that $\lambda-\mu$ is non-vanishing. In particular, we have a quadratic equation in $\frac{c}{\rho}$, which we can then solve to obtain

$$
\frac{c}{\rho}=\frac{-5 \pm \sqrt{D}}{8} \quad \text { where } \quad D=\frac{9 \lambda+7 \mu}{\lambda-\mu} .
$$

Now substituting (27) into (30) and (31), we get

$$
\lambda+\mu=2 \sqrt{\frac{\rho+c}{\rho+2 c}}\left(\frac{\rho}{\rho+c}-4\right)=-\frac{2(3+4 c / \rho)}{\sqrt{(1+2 c / \rho)(1+c / \rho)}} .
$$

Upon substituting (33) into the above and eliminating the square roots, we then obtain

$$
(\lambda+\mu)^{2} P(\lambda, \mu)=0
$$

From (32) we see that $\lambda+\mu$ vanishes if and only if $(3 \rho+4 c)(\rho+2 c)$ vanishes. Since this is not generically the case, the constraint (29) follows.

Remark 4.10 More generally, if we introduce the polynomial

$$
P_{\Lambda}(\lambda, \mu):=(\lambda+\mu)^{3} \mu+\frac{2 \Lambda}{3}\left(3 \lambda^{2}+18 \lambda \mu+11 \mu^{2}\right)+\frac{128 \Lambda^{2}}{9},
$$

then (12) and (13) imply

$$
P_{\Lambda}^{\prime}=-3 \mu P_{\Lambda}
$$

So the vanishing set of $P_{\Lambda}(\lambda, \mu)$ contains a flowline. When $\Lambda=-6$, this becomes the constraint (29).

Proposition 4.11 Any solution $(\lambda(t), \mu(t))$ of the Einstein equations (12)-(14) with negative Einstein constant $\Lambda$ satisfies the following constraint on each maximal domain of definition:

$$
\left(\frac{\mu^{2}+\lambda \mu+2 \Lambda}{\mu^{2}+2 \lambda \mu+4 \Lambda}\right)^{3} P_{\Lambda}(\lambda, \mu)=K,
$$

for some constant $K$. Furthermore, when $K=0$, the solution has to be one of the following:

- Stationary solutions at $\pm(2 \sqrt{-2 \Lambda / 3}, \sqrt{-2 \Lambda / 3})$.

- Solutions with maximal domains of definition $]-\infty, t_{0}[$ and $] t_{0},+\infty[$ which are given by

$$
\mu=\sqrt{-\frac{2 \Lambda}{3}} \frac{e^{\sqrt{-6 \Lambda}\left(t-t_{0}\right)} \pm 1}{e^{\sqrt{-6 \Lambda}\left(t-t_{0}\right)} \mp 1}, \quad \lambda=2 \sqrt{-\frac{2 \Lambda}{3}} \frac{e^{2 \sqrt{-6 \Lambda}\left(t-t_{0}\right)} \pm 4 e^{\sqrt{-6 \Lambda}\left(t-t_{0}\right)}+1}{e^{2 \sqrt{-6 \Lambda}\left(t-t_{0}\right)}-1} .
$$

- A solution with maximal domains of definition $]-\infty, t_{0}[$ and $] t_{0},+\infty[$ which is given by 


$$
\begin{gathered}
\lambda=\frac{\operatorname{sign}\left(t-t_{0}\right) 8 \sqrt{-2 \Lambda / 3}}{\sqrt{3+18 \sigma+11 \sigma^{2}-\sqrt{(1-\sigma)^{3}(9+7 \sigma)}}, \quad \mu=\lambda \sigma, \quad \text { where } 0<\sigma<1,} \\
\int_{0}^{\sigma} \frac{-8 \sqrt{3+18 u+11 u^{2}-\sqrt{(1-u)^{3}(9+7 u)}} d u}{(1-u)(1-5 u)(9+7 u)-3(1+3 u) \sqrt{(1-u)^{3}(9+7 u)}}=\sqrt{-\frac{2 \Lambda}{3}}\left|t-t_{0}\right| .
\end{gathered}
$$

- A solution with maximal domains of definition $]-\infty, t_{0}[$ and $] t_{0},+\infty[$ which is given by

$$
\begin{gathered}
\lambda=\frac{-\operatorname{sign}\left(t-t_{0}\right) 8 \sqrt{-2 \Lambda / 3}}{\sqrt{3+18 \sigma+11 \sigma^{2}+\sqrt{(1-\sigma)^{3}(9+7 \sigma)}}, \quad \mu=\lambda \sigma, \quad \text { where }-1<\sigma<\frac{1}{2},} \\
\int_{-1}^{\sigma} \frac{8 \sqrt{3+18 u+11 u^{2}+\sqrt{(1-u)^{3}(9+7 u)}} d u}{(1-u)(1-5 u)(9+7 u)+3(1+3 u) \sqrt{(1-u)^{3}(9+7 u)}}=\sqrt{-\frac{2 \Lambda}{3}}\left|t-t_{0}\right| .
\end{gathered}
$$

- Solutions defined for all $t \in \mathbb{R}$ given by

$$
\begin{aligned}
& \lambda=\frac{ \pm 8 \sqrt{-2 \Lambda / 3}}{\sqrt{3+18 \sigma+11 \sigma^{2}+\sqrt{(1-\sigma)^{3}(9+7 \sigma)}}, \quad \mu=\lambda \sigma, \quad \text { where } \frac{1}{2}<\sigma<1,} \\
& \int_{3 / 4}^{\sigma} \frac{\mp 8 \sqrt{3+18 u+11 u^{2}+\sqrt{(1-u)^{3}(9+7 u)}} d u}{(1-u)(1-5 u)(9+7 u)+3(1+3 u) \sqrt{(1-u)^{3}(9+7 u)}}=\sqrt{-\frac{2 \Lambda}{3}}\left(t-t_{0}\right) .
\end{aligned}
$$

With the exception of (39), all of them correspond to Riemannian metrics.

Proof Equations (12) and (13) imply

$$
\begin{gathered}
\left(\mu^{2}+2 \lambda \mu+4 \Lambda\right)^{\prime}=(\lambda-2 \mu)\left(\mu^{2}+2 \lambda \mu+4 \Lambda\right), \\
\left(\mu^{2}+\lambda \mu+2 \Lambda\right)^{\prime}=(\lambda-\mu)\left(\mu^{2}+\lambda \mu+2 \Lambda\right) .
\end{gathered}
$$

On the domain where $\mu^{2}+2 \lambda \mu+4 \Lambda$ is non-vanishing, (42) can be written as

$$
\frac{d\left(\mu^{2}+2 \lambda \mu+4 \Lambda\right)}{\mu^{2}+2 \lambda \mu+4 \Lambda}=\lambda d t-2 \mu d t
$$

Integrating and then exponentiating gives

$$
\mu^{2}+2 \lambda \mu+4 \Lambda=-\frac{4 k a}{b^{2}}
$$

where $k$ is a nonzero constant of integration. As we had noted in the proof of Proposition 4.5, given $\lambda=(\ln a)^{\prime}$ and $\mu=(\ln b)^{\prime}$, the functions $a$ and $b$ are determined only up to overall nonzero constant factors. Thus, the constant $k$ may be absorbed into this indeterminacy so that (14) is automatically satisfied, provided that $k>0$. So, we see that (14) is equivalent to the condition $\mu^{2}+2 \lambda \mu+4 \Lambda<0$.

Given that $\mu^{2}+2 \lambda \mu+4 \Lambda$ is nowhere vanishing, (36), (42), and (43) can be combined into a single equation: 


$$
\left(\frac{\left(\mu^{2}+\lambda \mu+2 \Lambda\right)^{\ell} P_{\Lambda}^{m}}{\left(\mu^{2}+2 \lambda \mu+4 \Lambda\right)^{n}}\right)^{\prime}=(\ell(\lambda-\mu)-3 m \mu-n(\lambda-2 \mu)) \frac{\left(\mu^{2}+\lambda \mu+2 \Lambda\right)^{\ell} P_{\Lambda}^{m}}{\left(\mu^{2}+2 \lambda \mu+4 \Lambda\right)^{n}}
$$

where $\ell, m, n$ are arbitrary non-negative integers. In particular, we see that the right-hand side vanishes for the choice $\ell=3, m=1, n=3$. The constraint (37) follows.

For $K=0$, we have either

$$
\mu^{2}+\lambda \mu+2 \Lambda=0 \quad \text { or } \quad P_{\Lambda}(\lambda, \mu)=0 .
$$

If $\mu^{2}+\lambda \mu+2 \Lambda=0$, then (13) becomes

$$
2 \mu^{\prime}=-3 \mu^{2}-2 \Lambda .
$$

If the right-hand side vanishes, then we obtain the stationary solutions

$$
\mu= \pm \sqrt{-\frac{2 \Lambda}{3}}, \quad \lambda=-\mu-\frac{2 \Lambda}{\mu}= \pm 2 \sqrt{-\frac{2 \Lambda}{3}} .
$$

Otherwise, we can separate the variables and integrate to obtain

$$
\log \left|\frac{\mu+\sqrt{-2 \Lambda / 3}}{\mu-\sqrt{-2 \Lambda / 3}}\right|=\sqrt{-6 \Lambda}\left(t-t_{0}\right)
$$

where $t_{0}$ is an integration constant. This gives the solutions

$$
\mu=\sqrt{-\frac{2 \Lambda}{3}} \frac{e^{\sqrt{-6 \Lambda}\left(t-t_{0}\right)} \pm 1}{e^{\sqrt{-6 \Lambda}\left(t-t_{0}\right)} \mp 1}, \quad \lambda=-\mu-\frac{2 \Lambda}{\mu}=2 \sqrt{-\frac{2 \Lambda}{3}} \frac{e^{2 \sqrt{-6 \Lambda}\left(t-t_{0}\right)} \pm 4 e^{\sqrt{-6 \Lambda}\left(t-t_{0}\right)}+1}{e^{2 \sqrt{-6 \Lambda}\left(t-t_{0}\right)}-1} .
$$

Both the upper and lower solutions are well-defined eveywhere except $t=t_{0}$. Moreover, the vanishing sets of $\mu^{2}+\lambda \mu+2 \Lambda$ and $\mu^{2}+2 \lambda \mu+4 \Lambda$ do not intersect. Thus, their maximal domains of definition are the open intervals $]-\infty, t_{0}[$ and $] t_{0},+\infty[$.

Now we consider the case $P_{\Lambda}(\lambda, \mu)=0$. Observe that at $\lambda=0$, we have

$$
P_{\Lambda}(0, \mu)=\left(\mu^{2}+\frac{11 \Lambda}{3}\right)^{2}+\frac{7 \Lambda^{2}}{9}>0 .
$$

Thus, it follows that $\lambda \neq 0$ on the vanishing set of $P_{\Lambda}$. We can therefore define $\sigma=\frac{\mu}{\lambda}$ and rewrite $P_{\Lambda}(\lambda, \mu)=0$ in terms of it as

$$
(1+\sigma)^{3} \sigma+\frac{2 \Lambda}{3 \lambda^{2}}\left(3+18 \sigma+11 \sigma^{2}\right)+\frac{128 \Lambda^{2}}{9 \lambda^{4}}=0 .
$$

This is quadratic in $\frac{1}{\lambda^{2}}$, so we can solve for it to obtain

$$
\frac{1}{\lambda^{2}}=\frac{-3\left(3+18 \sigma+11 \sigma^{2}\right) \pm 3 \sqrt{(1-\sigma)^{3}(9+7 \sigma)}}{128 \Lambda} .
$$

In order for the right-hand side to be real, we must have $-\frac{9}{7} \leq \sigma \leq 1$. Given that $\Lambda<0$, the upper solution is positive for $0<\sigma \leq 1$ and the lower solution is positive for $-1<\sigma \leq 1$. Note, however, that the case $\sigma=1$ has to be excluded as it implies $\mu=\lambda= \pm 2 \sqrt{-\Lambda / 3}$ and these are precisely the points where the vanishing sets of $P_{\Lambda}$ and $\mu^{2}+2 \lambda \mu+4 \Lambda$ intersect. 
To summarize, the allowed range for $\sigma$ in the upper solution is ]0, 1[, while that in the lower solution is ] $-1,1$. Moreover, for each such case, there are two solutions for $\lambda$, one positive and one negative.

Meanwhile, from (12) and (13), we can derive the following ode for $\sigma$ :

$$
2 \sigma^{\prime}=2 \sigma(1+\sigma)(2+\sigma) \lambda+\frac{4 \Lambda(1+3 \sigma)}{\lambda} .
$$

We can now obtain separable odes for $\sigma$ by substituting the solutions for $\lambda$ in (45) into the above:

$$
\begin{aligned}
& \mp \frac{8 \sqrt{3+18 \sigma+11 \sigma^{2}-\sqrt{(1-\sigma)^{3}(9+7 \sigma)}}}{\sqrt{-2 \Lambda / 3}} \sigma^{\prime} \\
& =(1-\sigma)(1-5 \sigma)(9+7 \sigma)-3(1+3 \sigma) \sqrt{(1-\sigma)^{3}(9+7 \sigma)} \\
& \mp \frac{8 \sqrt{3+18 \sigma+11 \sigma^{2}+\sqrt{(1-\sigma)^{3}(9+7 \sigma)}}}{\sqrt{-2 \Lambda / 3}} \sigma^{\prime} \\
& =(1-\sigma)(1-5 \sigma)(9+7 \sigma)+3(1+3 \sigma) \sqrt{(1-\sigma)^{3}(9+7 \sigma)} .
\end{aligned}
$$

The $\mp$ in the above refers to the choice of sign \pm of $\lambda$. The allowed range for $\sigma$ in (46) is $] 0,1$ [ while that in (47) is ] $-1,1$. The right-hand side in (46) is non-vanishing for all $\sigma$ in the allowed range ]0,1[, while the right-hand side in (47) vanishes only at $\sigma=\frac{1}{2}$ in the allowed range ] $-1,1[$. This corresponds to the stationary solution at $2 \mu=\lambda= \pm 2 \sqrt{-2 \Lambda / 3}$ that we already encountered in (44). On the complement of this, we can separate the variables and integrate to obtain

$$
\begin{aligned}
& \int_{\sigma_{0}}^{\sigma} \frac{8 \sqrt{3+18 u+11 u^{2}-\sqrt{(1-u)^{3}(9+7 u)}} d u}{(1-u)(1-5 u)(9+7 u)-3(1+3 u) \sqrt{(1-u)^{3}(9+7 u)}}=\mp \sqrt{-\frac{2 \Lambda}{3}}\left(t-t_{0}\right), \\
& \int_{\sigma_{0}}^{\sigma} \frac{8 \sqrt{3+18 u+11 u^{2}+\sqrt{(1-u)^{3}(9+7 u)}} d u}{(1-u)(1-5 u)(9+7 u)+3(1+3 u) \sqrt{(1-u)^{3}(9+7 u)}}=\mp \sqrt{-\frac{2 \Lambda}{3}}\left(t-t_{0}\right),
\end{aligned}
$$

where $u$ is a dummy integration variable, while $\sigma_{0}$ and $t_{0}$ are integration constants. The integration constants are redundant and the choice of $\sigma_{0}$ may be absorbed into the choice of $t_{0}$.

We now make the general observation that if the integrand $f(u)$ of a given integral $F(\sigma):=\int_{\sigma_{0}}^{\sigma} f(u) d u$ has the asymptotic behavior $f(u) \sim\left(u-u_{0}\right)^{\alpha}$ as $u \rightarrow u_{0}$ and is welldefined over the half-closed interval $\left\lfloor\sigma_{0}, u_{0}\left\lfloor\right.\right.$ (if $\sigma_{0}<u_{0}$ ) or $\left.\rfloor u_{0}, \sigma_{0}\right\rfloor$ (if $\sigma_{0}>u_{0}$ ), then $F(\sigma)$ converges in the limit $\sigma \rightarrow u_{0}$ when $\alpha>-1$ and diverges otherwise. For (48), the integrand has the asymptotic behavior 


$$
\begin{aligned}
& \frac{8 \sqrt{3+18 u+11 u^{2}-\sqrt{(1-u)^{3}(9+7 u)}}}{(1-u)(1-5 u)(9+7 u)-3(1+3 u) \sqrt{(1-u)^{3}(9+7 u)}} \sim-\frac{1}{\sqrt{3 u}} \quad \text { as } u \rightarrow 0, \\
& \frac{8 \sqrt{3+18 u+11 u^{2}-\sqrt{(1-u)^{3}(9+7 u)}}}{(1-u)(1-5 u)(9+7 u)-3(1+3 u) \sqrt{(1-u)^{3}(9+7 u)}} \sim-\frac{2 \sqrt{2}}{3 \sqrt{(1-u)^{3}}} \quad \text { as } u \rightarrow 1 .
\end{aligned}
$$

Thus, the integral is well-defined in the limit $\sigma \rightarrow 0$, so we can set $\sigma_{0}=0$. Then, as $\sigma \rightarrow 0$, we have $t \rightarrow t_{0}$, while as $\sigma \rightarrow 1$, we have $t \rightarrow \pm \infty$. For all other values of $\sigma$ between these two limits, the integral is well-defined. So, the maximal domains of definition of the upper and lower solutions are $] t_{0},+\infty[$ and $]-\infty, t_{0}[$, respectively. These can be combined into a single solution (39).

For (49), the integrand has the asymptotic behavior

$$
\begin{aligned}
& \frac{8 \sqrt{3+18 u+11 u^{2}+\sqrt{(1-u)^{3}(9+7 u)}}}{(1-u)(1-5 u)(9+7 u)+3(1+3 u) \sqrt{(1-u)^{3}(9+7 u)}} \sim-\frac{\sqrt{u+1}}{2} \quad \text { as } u \rightarrow-1, \\
& \frac{8 \sqrt{3+18 u+11 u^{2}+\sqrt{(1-u)^{3}(9+7 u)}}}{(1-u)(1-5 u)(9+7 u)+3(1+3 u) \sqrt{(1-u)^{3}(9+7 u)}} \sim-\frac{1}{\left(u-\frac{1}{2}\right)} \quad \text { as } u \rightarrow \frac{1}{2}, \\
& \frac{8 \sqrt{3+18 u+11 u^{2}+\sqrt{(1-u)^{3}(9+7 u)}}}{(1-u)(1-5 u)(9+7 u)+3(1+3 u) \sqrt{(1-u)^{3}(9+7 u)}} \sim \frac{2 \sqrt{2}}{3 \sqrt{(1-u)^{3}}} \quad \text { as } u \rightarrow 1 .
\end{aligned}
$$

The integral is ill-defined when $\sigma=\frac{1}{2}$ lies in the domain of integration. So we have two qualitatively different choices, namely $\sigma_{0}<\frac{1}{2}$ and $\sigma_{0}>\frac{1}{2}$. In the first case, since the integral is well-defined in the limit $\sigma \rightarrow-1$, we can set $\sigma_{0}=-1$. Then, as $\sigma \rightarrow-1$, we have $t \rightarrow t_{0}$, while as $\sigma \rightarrow \frac{1}{2}$ from below, we have $t \rightarrow \mp \infty$. For all other values of $\sigma$ between these two limits, the integral is well-defined. So, the maximal domains of definition of the upper and lower solutions are $]-\infty, t_{0}[$ and $] t_{0},+\infty[$, respectively. These can be combined into a single solution (40).

However, if $\sigma_{0}>\frac{1}{2}$, say $\sigma=\frac{3}{4}$, then as $\sigma \rightarrow \frac{1}{2}$ from above, we have $t \rightarrow \pm \infty$, while as $\sigma \rightarrow 1$, we have $t \rightarrow \mp \infty$. For all other values of $\sigma$ between these limits, the integral is welldefined, so we have two solutions defined for all $t \in \mathbb{R}$, namely (41).

It remains to check which of the solutions satisfy the sign constraint $\mu^{2}+2 \lambda \mu+4 \Lambda<0$ necessary for the metric to be positive definite. Note that the condition $\mu^{2}+\lambda \mu+2 \Lambda=0$ automatically implies

$$
\mu^{2}+2 \lambda \mu+4 \Lambda=2\left(\mu^{2}+\lambda \mu+2 \Lambda\right)-\mu^{2}=-\mu^{2}<0 .
$$

So the stationary solutions and the solution (38) satisfy the sign constraint.

For the rest of the solutions, we see using (45) that

$$
\sigma^{2}+2 \sigma+\frac{4 \Lambda}{\lambda^{2}}=\frac{(\sigma-9)(1-\sigma) \pm 3 \sqrt{(1-\sigma)^{3}(9+7 \sigma)}}{32}
$$

For $-1<\sigma<1$, the right-hand side is positive for the upper solution and negative for the lower solution. Multiplying by $\lambda^{2}$ throughout then tells us that (39) (which corresponds to 
the upper solution) does not satisfy the sign constraint, while (40) and (41) (which correspond to the lower solution) do satisfy the sign constraint.

Remark 4.12 For $\Lambda=-6$, the two stationary solutions are the solutions associated with the complex hyperbolic plane with holomorphic sectional curvature -4 , while the non-stationary solutions in (40) and (41) are the solutions associated with the one-loop deformed universal hypermultiplet.

The change of coordinates between $\sigma$ and $\rho$ may be deduced from (33) to be

$$
\frac{\rho}{c}=-8\left(5 \mp \sqrt{\frac{9+7 \sigma}{1-\sigma}}\right)^{-1} .
$$

This is well defined for $-1<\sigma<1$. Multiplying (31) by $\frac{d t}{d \sigma}$ and using the chain rule on the right-hand side gives us

$$
\mu \frac{d t}{d \sigma}=-\frac{1}{\rho}\left(\frac{\rho+4 c}{\rho+2 c}\right) \frac{d \rho}{d \sigma}
$$

Using the explicit expressions in (40) and (41), we find that

$$
\mu \frac{d t}{d \sigma}=\frac{-64 \sigma}{(1-\sigma)(1-5 \sigma)(9+7 \sigma)+3(1+3 \sigma) \sqrt{(1-\sigma)^{3}(9+7 \sigma)}} .
$$

Meanwhile, using (50), we find that

$$
-\frac{1}{\rho}\left(\frac{\rho+4 c}{\rho+2 c}\right) \frac{d \rho}{d \sigma}=\frac{-64 \sigma}{(1-\sigma)(1-5 \sigma)(9+7 \sigma) \pm 3(1+3 \sigma) \sqrt{(1-\sigma)^{3}(9+7 \sigma)}} .
$$

Thus, we see that (51) holds only for the upper solution

$$
\frac{\rho}{c}=-8\left(5-\sqrt{\frac{9+7 \sigma}{1-\sigma}}\right)^{-1}=-\frac{1-\sigma}{2(1-2 \sigma)}\left(5+\sqrt{\frac{9+7 \sigma}{1-\sigma}}\right) .
$$

When $\frac{1}{2}<\sigma<1$, we have $\frac{\rho}{c}>0$, while when $-1<\sigma<\frac{1}{2}$, we have $\frac{\rho}{c}<-2$. This is consistent with the fact that the one-loop deformed universal hypermultiplet metric is complete over the domain $\frac{\rho}{c}>0$ and incomplete over the domain $\frac{\rho}{c}<-2$.

In principle, for arbitrary values of $K$, the constraint (37) allows us to write $\lambda$ as an implicit function of $\mu$, which can then be used to turn (13) into a separable ode in $\mu$. However, as (37) amounts to a bivariate polynomial equation of degree 7, the implicit function cannot be expected to have a closed form in terms of radicals. Nevertheless, it is possible to make conclusions about the completeness of the solutions, as in the next theorem. From Sect. 4.2 and Myer's theorem we know that the Einstein constant $\Lambda$ of any complete solution of the Einstein equations (12)-(14) is necessarily negative. So we may as well assume $\Lambda=-6$.

Theorem 4.13 The complex hyperbolic metric (16) of constant holomorphic sectional curvature -4 and the one-loop deformed universal hypermultiplet metric with $\frac{\rho}{c}>0$ are the only complete solutions of the Einstein equations (12)-(14) for $\Lambda=-6$. 
Proof Suppose we have a complete solution $(\lambda(t), \mu(t))$ of (12)-(14). The solution is either bounded in both the limits $t \rightarrow \pm \infty$ or unbounded in at least one.

We first consider the bounded case. Fix a real number $k$ and define

$$
\begin{aligned}
& f(\lambda, \mu)=2(\lambda+k \mu) P_{\Lambda}(\lambda, \mu)^{2}, \\
& h(\lambda, \mu)=\lambda^{2}+(2+12 k) \mu^{2}+(18-3 k) \lambda \mu+(12-4 k) \Lambda .
\end{aligned}
$$

By (12), (13), and (36), we have

$$
f^{\prime}=-h P_{\Lambda}^{2}
$$

The function $h$ is strictly positive, and so $-h P_{\Lambda}^{2}$ is non-positive, for all $\lambda, \mu$ and all $\Lambda<0$ whenever $k$ satisfies

$$
3<k \leq \frac{26+6 \sqrt{10}}{3} .
$$

Thus, given that $k$ is in the above range, we have a monotonically decreasing function $f(\lambda(t), \mu(t))$ of $t$.

Any monotonically decreasing function of $t$ is either unbounded or has well-defined (finite) limits as $t \rightarrow \pm \infty$. Since our solution is assumed to be bounded, it has to be the latter case. In fact, the limiting value of $f$ must be one for which $f^{\prime}=-h P_{\Lambda}^{2}$ (and hence $P_{\Lambda}$ ) vanishes. Thus, we have a well-defined limit

$$
\lim _{t \rightarrow \pm \infty} P_{\Lambda}(\lambda(t), \mu(t))=0 .
$$

The constant $K$ in the constraint (37) is either zero or nonzero. We have already explicitly described the $K=0$ case in Proposition 4.11 and seen in Remark 4.12 that the only complete solutions for $\Lambda=-6$ correspond to precisely the complex hyperbolic metric with holomorphic sectional curvature -4 and the one-loop deformed universal hypermultiplet metric with $\frac{\rho}{c}>0$. So we may assume $K \neq 0$ now. Since the solution is bounded, this implies that

$$
\lim _{t \rightarrow \pm \infty}\left(\mu(t)^{2}+2 \lambda(t) \mu(t)+4 \Lambda\right)^{3}=\lim _{t \rightarrow \pm \infty} \frac{1}{K}\left(\mu(t)^{2}+\lambda(t) \mu(t)+2 \Lambda\right)^{3} P_{\Lambda}(\lambda(t), \mu(t))=0 .
$$

The vanishing sets of the polynomials $\mu^{2}+2 \lambda \mu+4 \Lambda$ and $P_{\Lambda}$ intersect in precisely the points $\pm\left(\frac{2 \sqrt{-\Lambda}}{\sqrt{3}}, \frac{2 \sqrt{-\Lambda}}{\sqrt{3}}\right)$. These can be checked to be fixed points of the first-order ode system (12) and (13). Moreover, $\left(\frac{2 \sqrt{-\Lambda}}{\sqrt{3}}, \frac{2 \sqrt{-\Lambda}}{\sqrt{3}}\right)$ is a stable fixed point, while $-\left(\frac{2 \sqrt{-\Lambda}}{\sqrt{3}}, \frac{2 \sqrt{-\Lambda}}{\sqrt{3}}\right)$ is an unstable fixed point. It thus follows that

$$
\lim _{t \rightarrow \pm \infty}(\lambda(t), \mu(t))= \pm\left(\frac{2 \sqrt{-\Lambda}}{\sqrt{3}}, \frac{2 \sqrt{-\Lambda}}{\sqrt{3}}\right) .
$$

Now, as the vanishing set of $\mu^{2}+\lambda \mu+2 \Lambda$ is a hyperbola, its complement in $\mathbb{R}^{2}$ consists of three connected components. The two fixed points $\pm\left(\frac{2 \sqrt{-\Lambda}}{\sqrt{3}}, \frac{2 \sqrt{-\Lambda}}{\sqrt{3}}\right)$ are on two different connected components; hence, any complete solution has to intersect the hyperbola $\mu^{2}+\lambda \mu+2 \Lambda=0$. But this is not possible since $K$ vanishes if $\mu^{2}+\lambda \mu+2 \Lambda$ vanishes, and we have assumed that $K \neq 0$. 
Next, we consider the case where the solution $(\lambda(t), \mu(t))$ is unbounded in at least one of the limits $t \rightarrow \pm \infty$. Without loss of generality, we may assume the solution is unbounded as $t \rightarrow+\infty$. Then there exists a sequence $t_{i} \rightarrow+\infty$ such that at least one of the following holds:

$$
\lim _{i \rightarrow \infty} \frac{1}{\lambda\left(t_{i}\right)}=\lim _{i \rightarrow \infty}\left(\frac{1}{\lambda\left(t_{i}\right)}\right)^{\prime}=0 \quad \text { or } \quad \lim _{i \rightarrow \infty} \frac{1}{\mu\left(t_{i}\right)}=\lim _{i \rightarrow \infty}\left(\frac{1}{\mu\left(t_{i}\right)}\right)^{\prime}=0 .
$$

Since the solution satisfies a polynomial constraint, by regarding it as the vanishing set of a polynomial on $\mathbb{R} \mathbb{P}^{2}$, we even see that $\lim _{t \rightarrow \infty} \frac{1}{\lambda(t)}=0$ or $\lim _{t \rightarrow \infty} \frac{1}{\mu(t)}=0$.

If we let $\sigma=\frac{\mu}{\lambda}$ and $v=\frac{\lambda}{\mu}$, then the constraint (37) may be rewritten in the following manner over suitable domains:

$$
\begin{aligned}
& \left(\frac{\sigma+1+2 \Lambda \lambda^{-2}}{\sigma+2+4 \Lambda \lambda^{-2}}\right)^{3}\left((1+\sigma)^{3} \sigma+\frac{2 \Lambda}{3 \lambda^{2}}\left(3+18 \sigma+11 \sigma^{2}\right)+\frac{128 \Lambda^{2}}{9 \lambda^{4}}\right)=\frac{K}{\lambda^{4}}, \\
& \left(\frac{1+v+2 \Lambda \mu^{-2}}{1+2 v+4 \Lambda \mu^{-2}}\right)^{3}\left((v+1)^{3}+\frac{2 \Lambda}{3 \mu^{2}}\left(3 \nu^{2}+18 v+11\right)+\frac{128 \Lambda^{2}}{9 \mu^{4}}\right)=\frac{K}{\mu^{4}}
\end{aligned}
$$

where $K$ is some constant. The above imply that in the limit $|\lambda| \rightarrow \infty$, we have either $\sigma=-1$ or $\sigma=0$, while in the limit $|\mu| \rightarrow \infty$, we have $\nu=-1$. Meanwhile (12) and (13) imply that

$$
\begin{aligned}
& \left(\frac{2}{\lambda}\right)^{\prime}=1+2 \sigma^{2}+6 \sigma+\frac{12 \Lambda}{\lambda^{2}}, \\
& \left(\frac{2}{\mu}\right)^{\prime}=-3 v-\frac{4 \Lambda}{\mu^{2}} .
\end{aligned}
$$

Thus, in the limit $|\lambda| \rightarrow \infty$, we have either $\left(\frac{2}{\lambda}\right)^{\prime} \rightarrow-3$ or $\left(\frac{2}{\lambda}\right)^{\prime} \rightarrow 1$, while in the limit $|\mu| \rightarrow \infty$, we have $\left(\frac{2}{\lambda}\right)^{\prime} \rightarrow 3$. This gives a contradiction.

Acknowledgements We thank Maciej Dunajski, Ángel Murcia, and Simon Salamon for useful comments.

Funding Open Access funding enabled and organized by Projekt DEAL. This work was supported by the German Science Foundation (DFG) under Germany's Excellence Strategy - EXC 2121 "Quantum Universe" -390833306.

Open Access This article is licensed under a Creative Commons Attribution 4.0 International License, which permits use, sharing, adaptation, distribution and reproduction in any medium or format, as long as you give appropriate credit to the original author(s) and the source, provide a link to the Creative Commons licence, and indicate if changes were made. The images or other third party material in this article are included in the article's Creative Commons licence, unless indicated otherwise in a credit line to the material. If material is not included in the article's Creative Commons licence and your intended use is not permitted by statutory regulation or exceeds the permitted use, you will need to obtain permission directly from the copyright holder. To view a copy of this licence, visit http://creativecommons.org/licenses/by/4.0/. 


\section{References}

1. Antoniadis, I., Minasian, R., Theisen, S., Vanhove, P.: String loop corrections to the universal hypermultiplet. Class. Quant. Grav. 20(23), 5079-5102 (2003)

2. Alekseevsky, D.V., Cortés, V., Dyckmanns, M., Mohaupt, T.: Quaternionic Kähler metrics associated with special Kähler manifolds. J. Geom. Phys. 92, 271-287 (2015)

3. Cortés, V., Saha, A.: Quarter-pinched Einstein metrics interpolating between real and complex hyperbolic metrics. Math. Z. 290, 155-166 (2018)

4. Cortés, V., Saha, A., Thung, D.: Symmetries of quaternionic Kähler manifolds with $S^{1}$-symmetry. Trans. London Math. Soc. 8(1), 95-119 (2021)

5. Dunajski, M., Hoegner, M.: SU(2) solutions to self-duality equations in eight dimensions. J. Geom. Phys. 62(8), 1747-1759 (2012)

6. Ellis, G.F.R., MacCallum, M.A.H.: A class of cosmological models. Commun. Math. Phys. 12, 108141 (1969)

7. Hein, H.-J., Sun, S., Viaclovsky, J., Zhang, R.: Nilpotent structures and collapsing Ricci-flat metrics on K3 surfaces. J. Amer. Math. Soc., to appear

8. Robles Llana, D., Saueressig, F., Vandoren, S.: String loop corrected hypermultiplet moduli spaces. J. High Energy Phys. 2006(03), 81 (2006)

9. Salamon, S.: Lie groups and special holonomy. Geometria Diferencial Unicamp, Geometry Webinar AmSur/AmSul 29, https://www.youtube.com/watch?v=ylixrP0jlPE

10. Wilson, E.N.: Isometry groups on homogeneous nilmanifolds. Geometriae Dedicata 12, 337-346 (1982)

Publisher's Note Springer Nature remains neutral with regard to jurisdictional claims in published maps and institutional affiliations. 